\title{
On an Optimal Choice of Pre-Perforated Tipping Paper and Porous Plug Wrap Paper
}

\author{
by \\ Bernhard Eitzinger \\ WFT Research, Fabrikstrasse 20, 4050 Traun, Austria
}

\section{SUMMARY}

To minimize the effect of permeability variations of tipping and plug wrap paper on the degree of filter ventilation and consequently on the smoke yields, a mathematical model for the flow through a layered structure of perforated tipping paper and porous plug wrap paper was derived. The output of the model was compared to experimental data and a very good agreement was found.

A statistical investigation revealed that measured permeability values of tipping and plug wrap papers are normally distributed. Furthermore, it was shown that the statistical distribution of the total permeability of the layered structure cannot be distinguished from a normal distribution at reasonable levels of statistical significance.

Based on these investigations and on the mathematical model, expressions for the expected value and the coefficient of variation of the total permeability were derived. It was shown that in spite of the non-linear model the difference between the exact expected total permeability and a simple estimate calculated from the expected permeabilities of tipping and plug wrap is sufficiently small, such that for all practical purposes this estimate can be used.

The coefficient of variation of the total permeability was then minimized. The solution of this optimisation problem delivered practically applicable design rules for the permeabilities of tipping and plug wrap papers. It was shown that the minimal coefficient of variation of the total permeability is lower than the coefficient of variation of the permeability of tipping and plug wrap paper. Typically an optimal design can be achieved by choosing the tipping paper permeability between $10 \%$ and $50 \%$ higher than the total permeability. A simple example demonstrated the usefulness of this approach. [Beitr. Tabakforsch. Int. 21 (2005) 358-367]

\section{ZUSAMMENFASSUNG}

Das Ziel dieser Untersuchungen ist es, den Einfluss von Schwankungen in der Permeabilität von Tipping- und Filterhüllpapieren auf den Filterventilationsgrad und damit auch auf die Abrauchwerte zu minimieren. Dazu wurde ein mathematisches Model abgeleitet, das die Strömung durch eine Schichtstruktur bestehend aus einem perforierten Tippingpapier und einem natürlich porösen Filterhüllpapier beschreibt. Die Vorhersagen dieses Modells wurden mit Messwerten verglichen, und eine sehr gute Übereinstimmung festgestellt.

In einer statistischen Untersuchung wurde festgestellt, dass die Permeabilitäten von Tipping- und Filterhüllpapieren im allgemeinen normalverteilt sind. Ebenso konnte gezeigt werden, dass auch die Wahrscheinlichkeitsverteilung der Gesamtpermeabilität der Schichtstruktur auf einem statistisch sinnvollen Signifikanzniveau nicht von einer Normalverteilung unterschieden werden kann.

Aufbauend auf diesen Untersuchungen wurden Ausdrücke für den Erwartungswert und den Variationskoeffizienten der Gesamtpermeabilität abgeleitet. Es zeigte sich, dass trotz des nichtlinearen Modells der Unterschied zwischen dem Erwartungswert der Gesamtpermeabilität und einem Schätzwert, berechnet aus den Erwartungswerten der Permeabilität von Tipping und Filterhüllpapier, so gering ist, dass er in allen praktischen Anwendungsfällen vernachlässigt werden darf.

Danach wurde der Variationskoeffizient der Gesamtpermeabilität minimiert. Das Ergebnis dieses Optimierungsproblems ist eine praktisch sehr gut anwendbare Richtlinie zur Bestimmung der Permeabilitäten von Tipping- und Filterhüllpapier. Es konnte gezeigt werden, dass der minimale Variationskoeffizient sogar niedriger ist als die Varia- 
tionskoeffizienten von Tipping- und Filterhüllpapier. In einem typischen optimalen Design liegt der Wert für die Permeabilität des Tippingpapiers etwa $10 \%$ bis $50 \%$ über der Gesamtpermeabilität. Die Nützlichkeit dieses Ansatzes wurde an einem einfachen Beispiel demonstriert. [Beitr. Tabakforsch. Int. 21 (2005) 358-367]

\section{RESUME}

Afin de minimiser l'influence des variations de la perméabilité du papier à cigarette et du papier de gainage du filtre sur le degré de ventilation du filtre ainsi que sur les rendements de la fumée, un modèle mathématique a été développé décrivant le flux traversant un papier à cigarette perforé et un papier de gainage poreux. Les résultats de ce modèle sont en bon accord avec les valeurs mesurées.

Une analyse statistique montre une distribution normale des valeurs de perméabilité du papier à cigarette et du papier de gainage. Cela permet de supposer que la distribution de la perméabilité totale du modèle mathématique ne peut pas être distinguée d'une distribution normale à un niveau significatif.

Sur la base de ces recherches et du modèle mathématique, des expressions pour la valeur attendue et du coefficient de variation de la perméabilité totale ont été déduites. Malgré un modèle non-linéaire, la différence entre la perméabilité totale attendue et la valeur estimative, résultant des perméabilités attendues du papier à cigarette et du papier de gainage est si minime qu'elle peut être négligée.

Le coefficient de variation de la perméabilité a donc été minimisé. Le résultat de ce problème d'optimisation permet une application assez pratique pour déterminer les perméabilités du papier à cigarette et du papier de gainage. Il a été démontré que le coefficient de variation normal est inférieur au coefficient de variation du papier à cigarette et du papier de gainage.

En choisissant la perméabilité du papier à cigarette entre $10 \%$ et $50 \%$ supérieure à la perméabilité totale, une désignation optimale est obtenue. Un exemple simple démontre l'avantage de cette méthode. [Beitr. Tabakforsch. Int. 21 (2005) 358-367]

\section{INTRODUCTION}

To achieve certain smoke yields many cigarette designs use a filter ventilation system which comprises a pre-perforated tipping paper and a porous plug wrap paper. During the cigarette design the tipping paper permeability and the plug wrap paper permeability need to be determined in order to get the desired degree of filter ventilation. There exist, however, infinitely many tipping and plug wrap paper combinations which yield the same filter ventilation and this degree of freedom can be used to pursue additional design goals.

In the present study, design rules will be derived from a mathematical model such that, in addition to getting the desired degree of filter ventilation, the coefficient of variation of the total permeability of the tipping/plug wrap combination is minimized. This will minimize the influence of permeability variations of tipping and plug wrap paper on the degree of filter ventilation and consequently reduce the variation in smoke yields.

In order to determine the necessary total permeability of the tipping/plug wrap combination, several mathematical models and computational schemes can be used, such as the ones described in (1-4), but for this study the total permeability will be assumed as given. The tipping paper permeability and the plug wrap paper permeability can then be calculated from the total permeability according to a model proposed in (5). In (5) the layered structure is modelled as two flow resistors with a linear pressure drop-flow relation switched in series. Our model will incorporate non-linear pressure drop-flow relations in the form of a power-law as proposed in (6) and also discussed in (7). The calculation of variances and of the coefficient of variation will be similar to (8).

First we will derive a mathematical model to calculate the total permeability of the layered structure from the permeabilities of tipping and plug wrap paper. The output of this model will be compared to measured data. After some considerations regarding the assumption of normally distributed measured values, the coefficient of variation of the total permeability will be minimized, resulting in practically applicable design rules.

\section{A MODEL FOR A LAYERED STRUCTURE}

In accordance with (6) and (7) the air flow through artificially perforated or naturally porous paper can be described by a power-law,

$$
\dot{V}=k A \Delta p^{n}
$$

where $\dot{V}$ denotes the volumetric flow, $\Delta p$ the pressure difference, $k$ the paper permeability and $A$ is the air permeable area. The exponent $n$ depends on the contributions of frictional and inertial forces to the total flow resistance (7). A more physically motivated model is given by $\Delta p=A\left(k_{1} \dot{V}+k_{2} \dot{V}^{2}\right)$, but both models fit experimental data very well (6). With respect to the complexity of the following algebraic computations, however, the power-law [1] has a definite advantage and will be used in this study.

We assume an isothermal and incompressible flow, such that the volumetric flow through both layers of the layered structure will be constant. The standardization of the temperature of equipment and samples used for air permeability measurements, as required in International Standard (ISO) 2965 (9), and the small contribution of friction forces to the heating of the gas flow justify the assumption of an isothermal flow. The assumption of incompressibility will introduce relative errors in the volumetric flow of approximately $1 \%$ (the ratio of pressure drop to air pressure), but this error can be eliminated during calibration and does not show up in the measured air permeability values. For a combination of tipping (TP) and plug wrap paper (PW) we then have

and

$$
\dot{V}=k_{\mathrm{TP}} A_{\mathrm{TP}} \Delta p_{\mathrm{TP}}^{n_{\mathrm{TP}}}
$$

$$
\dot{V}=k_{\mathrm{PW}} A_{\mathrm{PW}} \Delta p_{\mathrm{PW}}^{n_{\mathrm{PW}}}
$$

with the total pressure difference

$$
\Delta p=\Delta p_{\mathrm{TP}}+\Delta p_{\mathrm{PW}} .
$$


Unfortunately, in case $n_{\mathrm{TP}} \neq n_{\mathrm{PW}}$ no pressure drop-flow relation in the form of the power-law [1] can be derived for the layered structure such that the permeability $k$ is independent of the volumetric flow. Therefore, we will in the following assume that $n=n_{\mathrm{TP}}=n_{\mathrm{PW}}$ which is, of course, a simplification, but especially for electro-perforated tipping paper and naturally porous plug wrap paper the fit to experimental data is still very good. Typically, for electroperforated tipping paper we have $n_{\mathrm{TP}} \in[0.85,0.95]$ and for laser-perforated tipping we have $n_{\mathrm{TP}} \in[0.75,0.9]$ for medium and low permeability values. For plug wrap papers $n_{\mathrm{PW}}$ can be found in the interval $[0.6,0.8]$, with lower values applying to highly permeable papers. For most cigarette designs the pressure drop over the tipping paper will be much higher than over the plug wrap paper, because the tipping is the dominating flow resistance. Hence, the exponent $n$ assigned to the layered structure will match that of the tipping paper rather closely. This may explain why a good agreement with experimental data can be found in spite of the assumption $n=n_{\mathrm{TP}}=n_{\mathrm{PW}}$. Almost always we will have $n_{\mathrm{PW}}<n_{\mathrm{TP}}$ and it may be conjectured that a fit of measured volumetric flows through the layered structure will deliver an exponent $n \in\left[n_{\mathrm{PW}}, n_{\mathrm{TP}}\right]$.

From Eqns. [2] to [4] the total flow through the layered structure can be easily calculated by

$$
\dot{V}=\frac{k_{\mathrm{TP}} A_{\mathrm{TP}} k_{\mathrm{PW}} A_{\mathrm{PW}}}{\left(\left(k_{\mathrm{TP}} A_{\mathrm{TP}}\right)^{1 / n}+\left(k_{\mathrm{PW}} A_{\mathrm{PW}}\right)^{1 / n}\right)^{n}} \Delta p^{n} .
$$

We assume that $A_{\mathrm{PW}}=A_{\mathrm{TP}}$ such that the total permeability

$$
k_{\mathrm{tot}}=f\left(k_{\mathrm{TP}}, k_{\mathrm{PW}}\right)=\frac{k_{\mathrm{TP}} k_{\mathrm{PW}}}{\left(k_{\mathrm{TP}}^{1 / n}+k_{\mathrm{PW}}^{1 / n}\right)^{n}}
$$

can be assigned to the layered structure.

For a given total permeability and a known tipping paper permeability the plug wrap paper permeability can be calculated by

$$
k_{\mathrm{PW}}=k_{\mathrm{TP}}\left(k_{\mathrm{TP}}^{1 / n} k_{\mathrm{tot}}^{-1 / n}-1\right)^{-n}
$$

which can simply be proven by back-substituting into Eqn. [5]. Eqn.[6] will be needed later on.

While Eqn. [5] is rather simple, some words are necessary how the permeability values in Eqn. [5] are related to measurements of the air permeability by the standard method described in ISO 2965 (9). Let $k_{\mathrm{TP}, m}$ and $k_{\mathrm{PW}, m}$ be the permeabilities of tipping and plug wrap paper, respectively, as measured according to (9). Let $w$ be the perforation width. As the total area used in the permeability measurement of a perforated tipping paper is $A_{m}=20 \times 10 \mathrm{~mm}^{2}$, but the air permeable area is only $A_{\mathrm{TP}, m}=20 \times w \mathrm{~mm}^{2}$ we can calculate the local air permeability as needed in Eqn. [5] by

$$
k_{\mathrm{TP}}=\frac{A_{m}}{A_{\mathrm{TP}, m}} k_{\mathrm{TP}, m} .
$$

This equation works well for electro-perforated tipping paper, but for laser-perforated paper, which has a series of $N$ parallel perforation tracks, we can still use this equation with $w=0.5 \cdot N \mathrm{~mm}$, which on the one hand gives a good agreement with experimental data and on the other hand reflects a typical spacing of $0.5 \mathrm{~mm}$ between the perforation tracks. For the plug wrap paper no such correction is necessary, because the entire $20 \times 10 \mathrm{~mm}^{2}$ are air permeable. Overall we can calculate the total permeability $k_{\text {tot } m}$ as measured by the method in (9) by

$$
\begin{aligned}
k_{\mathrm{tot}, m} & =\frac{A_{\mathrm{TP}, m}}{A_{m}} \frac{\left(\frac{A_{m}}{A_{\mathrm{TP}, m}} k_{\mathrm{TP}, m}\right) k_{\mathrm{PW}, m}}{\left(\left(\frac{A_{m}}{A_{\mathrm{TP}, m}} k_{\mathrm{TP}, m}\right)^{1 / n}+k_{\mathrm{PW}, m}^{1 / n}\right)^{n}} \\
& =\frac{k_{\mathrm{TP}, m} k_{\mathrm{PW}, m}}{\left(\left(\frac{A_{m}}{A_{\mathrm{TP}, m}} k_{\mathrm{TP}, m}\right)^{1 / n}+k_{\mathrm{PW}, m}^{1 / n}\right)^{n}}
\end{aligned}
$$

It has to be noted that the model proposed in Eqn. [5] contains some simplifications. In (10), for example, it was shown, that the permeability depends on the direction of the air flow. This is not reflected in our model, because it is completely symmetric with respect to swapping $k_{\mathrm{TP}}$ and $k_{\mathrm{PW}}$. More importantly, on a cigarette the tipping paper and the plug wrap paper will be separated by the adhesive layer. Therefore a larger area of the plug wrap paper becomes available for the air flow, so that its flow resistance is reduced. From this point of view, the above assumption $A_{\mathrm{TP}}=A_{\mathrm{PW}}$ is not justified. In the experimental permeability measurements described below, however, the papers were simply stacked upon each other, so that no adhesive layer exists. Consequently, we assume $A_{\mathrm{TP}}=A_{\mathrm{PW}}$.

\section{VERIFICATION OF THE MODEL}

In the following we will frequently make use of the CORESTA unit $\mathrm{CU}=\mathrm{cm}^{3} /\left(\mathrm{cm}^{2} \cdot \mathrm{min} \cdot \mathrm{kPa}\right)$ as a unit for the air permeability.

To verify Eqn. [5] its predictions were compared to measured data. Electro-perforated tipping papers with permeabilities between 100 and $600 \mathrm{CU}$ and perforation widths of either 3 or $4 \mathrm{~mm}$ were combined with porous plug wrap papers with permeabilities from $2400 \mathrm{CU}$ up to $24000 \mathrm{CU}$. Overall, 35 combinations were measured with a Borgwaldt A10 according to ISO 2965, with 30 measurements on different positions per tipping/plug wrap combination. The two papers were simply stacked upon each other such that during the measurement the air flow occurred in the same direction as it will on a cigarette during puffing. The permeability was also calculated by Eqn. [7], with $n=0.87$ providing the best agreement with measured values. This value is also within the typical range of exponents for electro-perforated tipping paper. A linear regression of measured and calculated values gives an $R^{2}$ of 0.993 and a slope of the regression line of 1.0014, which means that calculated and measured values fit exceptionally well. The comparison for electro-perforated tipping paper is shown in Figure 1.

For laser-perforated tipping paper a permeability range from 250 to $1400 \mathrm{CU}$ was chosen with 1 to 6 perforation tracks. The range of plug wrap papers was again set to 2400 to $24000 \mathrm{CU}$. The permeability of the layered structure was measured 30 times on different positions for 38 tip- 


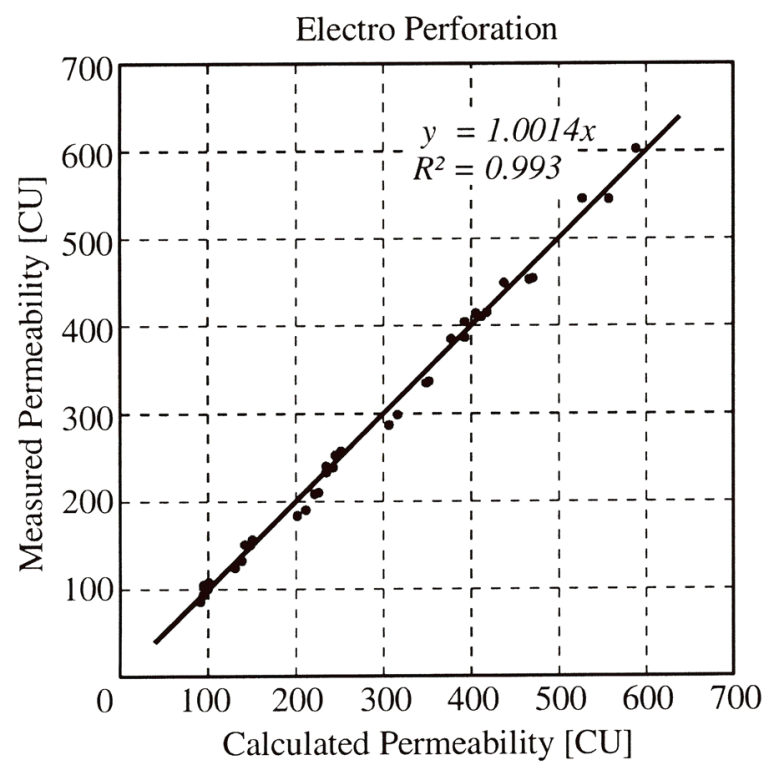

Figure 1. A comparison of calculated and measured permeability values of a layered structure of electro-perforated tipping paper and porous plug wrap paper. Each dot is the mean value of 30 measurements.

ping/plug wrap combinations. We chose $n=0.83$ to optimise the fit to measured values. A linear regression of measured and calculated values gives an $R^{2}$ of 0.948 and a slope of the regression line of 1.0015, as shown in Figure 2. This is still a good agreement but not as good as for the electro-perforated tipping paper. The deviation might be caused by the assumption that the exponents in Eqns. [2] and [3] are identical for both papers, or by the variation of the distance between the individual perforation tracks, which depends on the number of tracks.

Nonetheless the model fits sufficiently well to experimental data to be used in the following analysis.

\section{STATISTICAL CONSIDERATIONS}

The goal of this section is to determine typical variances of measured permeability values of tipping and plug wrap papers. Additionally, we will check, whether the measured values are normally distributed. While a normal distribution is not necessary for the following analysis, it has the advantage that it can be completely characterized by its mean value and variance.

To this end the permeability of an electro-perforated and of a laser-perforated tipping paper was measured at 1000 different but regularly spaced positions according to ISO 2965. By the same method two porous plug wrap papers
Laser Perforation

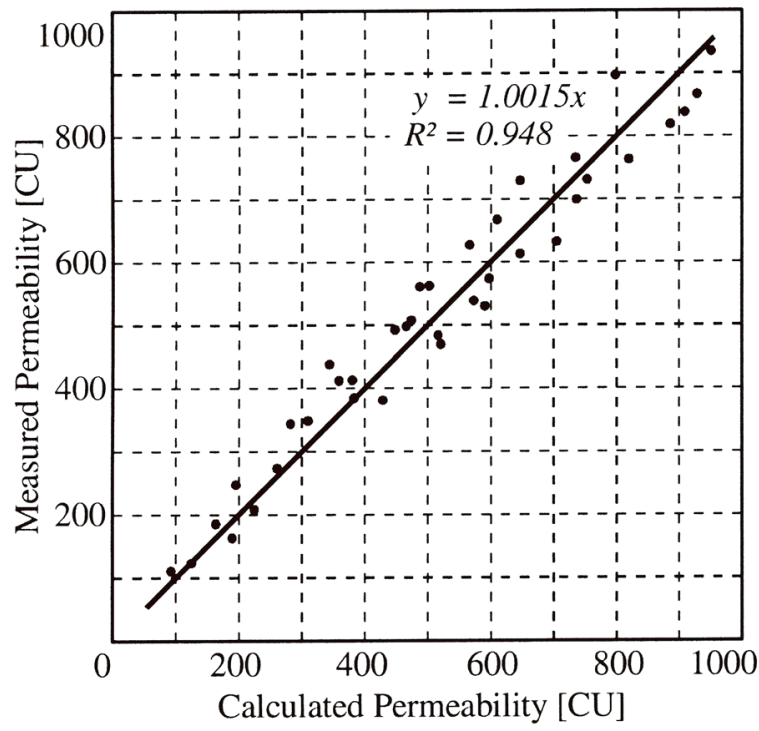

Figure 2. A comparison of calculated and measured permeability values of a layered structure of laser-perforated tipping paper and porous plug wrap paper. Each dot is the mean value of 30 measurements.

were measured at 2000 positions each. The results are shown in Table 1. As can be seen, typical coefficients of variation for perforated tipping papers are between $3 \%$ and $5 \%$, while for porous plug wrap papers they are between $7 \%$ and $10 \%$. On most perforation machines, the tipping paper permeability is measured on-line, usually by optical methods. The measured value is used to adjust the power of the perforation unit, which reduces the variation of the permeability compared to naturally porous paper and explains the observed difference in the coefficients of variation. It is also in agreement with practical experience that the coefficient of variation is lower for laser-perforated paper than for electroperforation, because for the laser-perforation the holes are regularly spaced and vary less in size. In general the coefficients of variation are specific to the process and do not vary much with the permeability, therefore they will be assumed as constant in the following investigation.

Just as with the permeability, certain corrections need to be made to account for the difference between the measurement and the real application on the cigarette. For the tipping paper an area of $A_{\mathrm{TP}, m}=20 \times w \mathrm{~mm}^{2}$ is air permeable during the measurement, but on a cigarette with a circumference of approximately $25 \mathrm{~mm}$ and $2 \mathrm{~mm}$ overlap an area of $A_{\mathrm{TP}, m}=23 \times \mathrm{w} \mathrm{mm}^{2}$ is available for the ventilation air flow. This reduces the coefficient of variation from its measured value $C_{\mathrm{TP}, m}$ to its value on the cigarette (numerical values for $w=3 \mathrm{~mm}$ )

Table 1. Statistical properties of measured permeabilities of electro-perforated and laser-perforated tipping papers (TP) and porous plug wrap papers (PW). The measured values are normally distributed if the statistic $T$ is less than the critical value.

\begin{tabular}{l|c|c|c|c|c|c}
\hline Paper & $\begin{array}{c}\text { Number of } \\
\text { values }\end{array}$ & $\begin{array}{c}\text { Mean value } \\
{[\mathrm{CU}]}\end{array}$ & $\begin{array}{c}\text { Standard } \\
\text { deviation [CU] }\end{array}$ & $\begin{array}{c}\text { Coefficient } \\
\text { of variation [\%] }\end{array}$ & $\begin{array}{c}\text { Statistic }\left(\chi^{2}\right) \\
20 \text { classes }\end{array}$ & $\begin{array}{c}\text { Critical value at 95\% } \\
\left(\chi_{17,0.95}^{2}\right)\end{array}$ \\
\hline TP electro & 1000 & 186.3 & 8.69 & 4.67 & 18.45 & 27.59 \\
TP laser & 1000 & 631.6 & 20.88 & 3.31 & 9.02 & 27.59 \\
PW A & 2000 & 5657.1 & 541.3 & 9.57 & 17.83 & 27.59 \\
PW B & 2000 & 11218.3 & 845.5 & 7.54 & 27.51 & 27.59 \\
\hline
\end{tabular}




$$
C_{\mathrm{TP}}=\sqrt{\frac{A_{\mathrm{TP}, m}}{A_{\mathrm{TP}}}} C_{\mathrm{TP}, m}=0.932 \cdot C_{\mathrm{TP}, m} .
$$

The same consideration holds for the plug wrap paper, with $A_{\mathrm{PW}, m}=20 \times 10 \mathrm{~mm}^{2}$ and $A_{\mathrm{PW}}=A_{\mathrm{TP}}$, such that

$$
C_{\mathrm{PW}}=\sqrt{\frac{A_{\mathrm{PW}, m}}{A_{\mathrm{PW}}}} C_{\mathrm{PW}, m}=1.702 \cdot C_{\mathrm{PW}, m}
$$

Eqns. [8] and [9] apply under certain rather general conditions and are proven in the Appendix.

Therefore, we have typically $C_{\mathrm{TP}} \in[0.03,0.05]$ and $C_{\mathrm{PW}} \in$ $[0.12,0.17]$. It has to be noted, of course, that the coefficient of variation determined by the methods above also contains the variation inherent to the measurement equipment and to the experimental procedure.

Whether the measured data are normally distributed was checked by the $\chi^{2}$-test (11). The data were divided into 20 classes and the statistic $\chi^{2}$ was calculated and compared to the critical value. The hypotheses of a normal distribution cannot be rejected, if the statistic is smaller than the critical value. The results are shown in Table 1.

The permeability values of all papers are normally distributed at the $95 \%$ confidence level, although the case with plug wrap B is rather close. Nonetheless we can safely assume that the data are normally distributed.

The total permeability will not be normally distributed in a strict mathematical sense, because the flow model [5] is non-linear. To assess the extent of the deviation from normality, the Kolmogorov-Smirnov-test was performed on the permeability values of the 35 electro-perforated and the 38 laser-perforated tipping/plug wrap samples described in the previous section. In contrast to the $\chi^{2}$-test the Kolmogorov-Smirnov-test is more appropriate for the small sample size of 30 values. In all 73 cases the assumption of a normal distribution could not be rejected at the $95 \%$ or $99 \%$ confidence level.

\section{MINIMIZATION OF THE VARIANCE}

As the total permeability is in good approximation normally distributed, all we have to do is to calculate its expected value (mean value) and its variance for given mean values and variances of the tipping and plug wrap paper permeability. This is our next goal and we will start with the expected value.

\section{Expected value}

In the following $E(K)$ and $\bar{K}$ will denote the expected value of the random variable $K$ and $V(K)$ will denote its variance. The coefficient of variation is defined by $C=\sqrt{V(K)} / E(K)$. Let $K_{\mathrm{TP}}, K_{\mathrm{PW}}$ and $K_{\mathrm{tot}}$ be the tipping permeability, plug wrap paper permeability and total permeability, respectively. These random variables are related to each other by Eqn. [5]. The goal of this section is to derive an error estimate for the approximation of the expected total permeability $E\left(K_{\text {tot }}\right)=E\left(f\left(K_{\mathrm{TP}}, K_{\mathrm{PW}}\right)\right)$ by $\tilde{K}_{\text {tot }}=f\left(E\left(K_{\mathrm{TP}}\right), E\left(K_{\mathrm{PW}}\right)\right)$. We decompose the random variables by $K=E(K)+\Delta K$ and consequently $E(\Delta K)=0$.We obtain

$$
\begin{aligned}
K_{\text {tot }} & =f\left(K_{\mathrm{TP}}, K_{\mathrm{PW}}\right) \\
& =\frac{\left(\bar{K}_{\mathrm{TP}}+\Delta K_{\mathrm{TP}}\right)\left(\bar{K}_{\mathrm{PW}}+\Delta K_{\mathrm{PW}}\right)}{\left(\left(\bar{K}_{\mathrm{TP}}+\Delta K_{\mathrm{TP}}\right)^{1 / n}+\left(\bar{K}_{\mathrm{PW}}+\Delta K_{\mathrm{PW}}\right)^{1 / n}\right)^{n}} .
\end{aligned}
$$

We assume that $\Delta K_{\mathrm{TP}}$ and $\Delta K_{\mathrm{PW}}$ are small and expand $f$ in a Taylor series up to order 2 , denoted by $\tilde{f}_{2}$, where the derivatives are evaluated at the expected values. The relative error in the expected value due to this approximation is below $0.03 \%$. A derivation of this limit can be found in the Appendix.

$$
\begin{aligned}
\tilde{f}_{2}\left(K_{\mathrm{TP}}, K_{\mathrm{PW}}\right)= & f\left(\bar{K}_{\mathrm{TP}}, \bar{K}_{\mathrm{PW}}\right)+\frac{\partial f}{\partial K_{\mathrm{TP}}} \Delta K_{\mathrm{TP}} \\
& +\frac{\partial f}{\partial K_{\mathrm{PW}}} \Delta K_{\mathrm{PW}}+\frac{\partial^{2} f}{\partial K_{\mathrm{TP}} \partial K_{\mathrm{PW}}} \Delta K_{\mathrm{TP}} \Delta K_{\mathrm{PW}} \\
& +\frac{1}{2} \frac{\partial^{2} f}{\partial K_{\mathrm{TP}}^{2}} \Delta K_{\mathrm{TP}}^{2}+\frac{1}{2} \frac{\partial^{2} f}{\partial K_{\mathrm{PW}}^{2}} \Delta K_{\mathrm{PW}}^{2}
\end{aligned}
$$

Then we take the expected value and simplify the expression by $E\left(\Delta K_{\mathrm{TP}}\right)=0, E\left(\Delta K_{\mathrm{PW}}\right)=0$, and $E\left(\Delta K_{\mathrm{TP}} \Delta K_{\mathrm{PW}}\right)=0$. The last equality follows from the independence of tipping and plug wrap permeability. Furthermore we notice that $E\left(\Delta K_{\mathrm{TP}}^{2}\right)=V\left(\Delta K_{\mathrm{TP}}\right)=V\left(K_{\mathrm{TP}}\right)$ which likewise holds for the plug wrap paper permeability.

$$
E\left(\tilde{f}_{2}\right)=\underbrace{f\left(\bar{K}_{\mathrm{TP}}, \bar{K}_{\mathrm{PW}}\right)}_{\tilde{K}_{\mathrm{tot}}}+\frac{1}{2} \frac{\partial^{2} f}{\partial K_{\mathrm{TP}}^{2}} V\left(K_{\mathrm{TP}}\right)+\frac{1}{2} \frac{\partial^{2} f}{\partial K_{\mathrm{PW}}^{2}} V\left(K_{\mathrm{PW}}\right)
$$

As is no surprise for non-linear functions, the function evaluated at the expected values of its arguments differs from the expected function value. The second derivatives lead to somewhat lengthy expressions which can be significantly simplified. The complete derivation is found in the appendix, we will only state the result (Eqn. [19]).

$$
\begin{aligned}
E\left(\tilde{f}_{2}\right)= & \tilde{K}_{\mathrm{tot}}-\frac{1}{2} \frac{1+n}{n} \\
& \left(\frac{\tilde{K}_{\mathrm{tot}}^{1+2 / n}}{\bar{K}_{\mathrm{TP}}^{2+1 / n} \bar{K}_{\mathrm{PW}}^{1 / n}} V\left(K_{\mathrm{TP}}\right)+\frac{\tilde{K}_{\mathrm{tot}}^{1+2 / n}}{\bar{K}_{\mathrm{TP}}^{1 / n} K_{\mathrm{PW}}^{2+1 / n}} V\left(K_{\mathrm{PW}}\right)\right)
\end{aligned}
$$

Now we substitute the coefficient of variation $V\left(K_{\mathrm{TP}}\right)=\bar{K}_{\mathrm{TP}}^{2} C_{\mathrm{TP}}^{2}$ for the variance, likewise for the plug wrap paper, and obtain

$$
E\left(\tilde{f}_{2}\right)=\tilde{K}_{\text {tot }}-\frac{1+n}{2 n} \frac{\tilde{K}_{\text {tot }}^{1+2 / n}}{\bar{K}_{\mathrm{TP}}^{1 / n} \bar{K}_{\mathrm{PW}}^{1 / n}}\left(C_{\mathrm{TP}}^{2}+C_{\mathrm{PW}}^{2}\right) .
$$

The relative error between the approximation of the expected total permeability $E\left(\tilde{f}_{2}\left(K_{\mathrm{TP}}, K_{\mathrm{PW}}\right)\right)$ and its estimated value $\tilde{K}_{\text {tot }}=f\left(E\left(K_{\mathrm{TP}}\right), E\left(K_{\mathrm{PW}}\right)\right)$ can be expressed as

$$
\frac{E\left(\tilde{f}_{2}\right)-\tilde{K}_{\text {tot }}}{\tilde{K}_{\text {tot }}}=-\frac{1+n}{2 n} \frac{\tilde{K}_{\text {tot }}^{2 / n}}{\bar{K}_{\mathrm{TP}}^{1 / n} \bar{K}_{\mathrm{PW}}^{1 / n}}\left(C_{\mathrm{TP}}^{2}+C_{\mathrm{PW}}^{2}\right) .
$$


To give an upper bound on the relative error, we notice by inequality [20] that $\tilde{K}_{\text {tot }}<\bar{K}_{\mathrm{TP}}$ and $\tilde{K}_{\text {tot }}<\bar{K}_{\mathrm{PW}}$. In typical cigarette designs we have $K_{\text {tot }} \approx \bar{K}_{\mathrm{TP}}$ and $K_{\mathrm{PW}}>5 \cdot \bar{K}_{\mathrm{TP}}$. Choosing $n=1, C_{\mathrm{TP}}=0.05$ and $C_{\mathrm{PW}}=0.17$, which is the worst case, and setting $\bar{K}_{\mathrm{TP}}=\tilde{K}_{\text {tot }}$ and $\bar{K}_{\mathrm{PW}}=5 \cdot \tilde{K}_{\text {tot }}$ we obtain

$$
-0.00628<\frac{E\left(\tilde{f}_{2}\right)-\tilde{K}_{\text {tot }}}{\tilde{K}_{\text {tot }}} \leq 0 .
$$

Taking the error due to the approximation by the Taylor series into account we finally get

$$
-0.00658<\frac{E\left(K_{\text {tot }}\right)-\tilde{K}_{\text {tot }}}{\tilde{K}_{\text {tot }}} \leq 0.0003 .
$$

Therefore, by approximating the true expected value for the total permeability with its simple estimate by evaluating Eqn. [5] at the expected values for tipping and plug wrap paper permeability, we make an error which is in magnitude usually less than $0.66 \%$.

\section{Coefficient of variation}

To calculate the variance of the total permeability, we start again with a Taylor series expansion $\tilde{f}_{1}$ of $f$, this time only up to order 1 . The relative errors will now be larger, but they are usually still less than $0.44 \%$. A detailed derivation of the error estimate can be found in the Appendix.

$$
\tilde{f}_{1}=\tilde{K}_{\mathrm{tot}}+\frac{\partial f}{\partial K_{\mathrm{TP}}} \Delta K_{\mathrm{TP}}+\frac{\partial f}{\partial K_{\mathrm{PW}}} \Delta K_{\mathrm{PW}}
$$

Due to the independence of $\Delta K_{\mathrm{TP}}$ and $\Delta K_{\mathrm{PW}}$ the following rules for variances apply:

$V\left(\Delta K_{\mathrm{TP}}+\Delta K_{\mathrm{PW}}\right)=V\left(K_{\mathrm{TP}}\right)+V\left(\Delta K_{\mathrm{PW}}\right)$ and

$V(a \Delta K)=a^{2} V(\Delta K)$. Hence, taking the variance we obtain

$$
V\left(\tilde{f}_{1}\right)=\left(\frac{\partial f}{\partial K_{\mathrm{TP}}}\right)^{2} V\left(\Delta K_{\mathrm{TP}}\right)+\left(\frac{\partial f}{\partial K_{\mathrm{PW}}}\right)^{2} V\left(\Delta K_{\mathrm{PW}}\right) .
$$

We notice that $V\left(\Delta K_{\mathrm{TP}}\right)=V\left(K_{\mathrm{TP}}\right)$ and we expand the derivatives by Eqn. [17].

$$
V\left(\tilde{f}_{1}\right)=\frac{1}{\left(\bar{K}_{\mathrm{TP}}^{1 / n}+\bar{K}_{\mathrm{PW}}^{1 / n}\right)^{2 n+2}}\left(\bar{K}_{\mathrm{PW}}^{2+2 / n} V\left(K_{\mathrm{TP}}\right)+\bar{K}_{\mathrm{TP}}^{2+2 / n} V\left(K_{\mathrm{PW}}\right)\right)
$$

Next we replace the variances by their corresponding coefficients of variation.

$$
V\left(\tilde{f}_{1}\right)=\frac{\bar{K}_{\mathrm{TP}}^{2}+\bar{K}_{\mathrm{PW}}^{2}}{\left(\bar{K}_{\mathrm{TP}}^{1 / n}+\bar{K}_{\mathrm{PW}}^{1 / n}\right)^{2 n+2}}\left(\bar{K}_{\mathrm{PW}}^{2 / n} C_{\mathrm{TP}}^{2}+\bar{K}_{\mathrm{TP}}^{2 / n} C_{\mathrm{PW}}^{2}\right)
$$

Due to the error estimates for expected value and variance derived above we may, as a good approximation, set $V\left(\tilde{f}_{1}\right)=V\left(K_{\text {tot }}\right)=C_{\text {tot }}^{2} E^{2}\left(K_{\text {tot }}\right)=C_{\text {tot }}^{2} \tilde{K}_{\text {tot }}^{2}$, which allows for some simplifications.

$$
\begin{aligned}
C_{\text {tot }}^{2} & =\underbrace{\frac{1}{\tilde{K}_{\text {tot }}^{2}} \frac{\bar{K}_{\mathrm{TP}}^{2} \bar{K}_{\mathrm{PW}}^{2}}{\left(\bar{K}_{\mathrm{TP}}^{1 / n}+\bar{K}_{\mathrm{PW}}^{1 / n}\right)^{2 n+2}}}_{\text {note equation [5] }}\left(\bar{K}_{\mathrm{PW}}^{2 / n} C_{\mathrm{TP}}^{2}+\bar{K}_{\mathrm{TP}}^{2 / n} C_{\mathrm{PW}}^{2}\right) \\
= & \frac{\bar{K}_{\mathrm{PW}}^{2 / n} C_{\mathrm{TP}}^{2}+\bar{K}_{\mathrm{TP}}^{2 / n} C_{\mathrm{PW}}^{2}}{\left(\bar{K}_{\mathrm{TP}}^{1 / n}+\bar{K}_{\mathrm{PW}}^{1 / n}\right)^{2}} .
\end{aligned}
$$

Now we have expressed the squared coefficient of variation as a function of the expected tipping and plug wrap paper permeability. To minimize the coefficient of variation for a given total permeability, we have to eliminate the plug wrap paper permeability according to Eqn. [6], because for a given total permeability it cannot be chosen independently of the tipping paper permeability. So we substitute

$$
\bar{K}_{\mathrm{PW}}=\bar{K}_{\mathrm{TP}}\left(\left(\frac{\bar{K}_{\mathrm{TP}}}{\tilde{K}_{\mathrm{tot}}}\right)^{1 / n}-1\right)^{-n}
$$

into Eqn. [11]. After some lengthy but straightforward algebraic manipulations, which were carried out with the computer algebra software package MAPLE, we finally obtain the simple expression

$$
C_{\mathrm{tot}}^{2}=\frac{\tilde{K}_{\mathrm{tot}}^{2 / n}}{\bar{K}_{\mathrm{TP}}^{2 / n}} C_{\mathrm{TP}}^{2}+\left(1-\frac{\tilde{K}_{\mathrm{tot}}^{1 / n}}{\bar{K}_{\mathrm{TP}}^{1 / n}}\right)^{2} C_{\mathrm{PW}}^{2}
$$

Now we can start to minimize the coefficient of variation of the total permeability.

\section{Solution of the minimization problem}

We introduce the ratio $\alpha$ of the tipping paper permeability to the total permeability, $\alpha=\bar{K}_{\mathrm{TP}} / \tilde{K}_{\text {tot }}$, substitute this into Eqn. [12] and solve the minimization problem

$$
\frac{\partial C_{\mathrm{tot}}}{\partial \alpha}=0
$$

After simple algebraic transformations we get the solution

$$
\alpha_{\min }=\left(\frac{C_{\mathrm{TP}}^{2}+C_{\mathrm{PW}}^{2}}{C_{\mathrm{PW}}^{2}}\right)^{n} .
$$

For a given total permeability we can therefore compute the tipping paper permeability for the minimal coefficient of variation by

$$
\bar{K}_{\mathrm{TP}, \min }=\tilde{K}_{\mathrm{tot}}\left(\frac{C_{\mathrm{TP}}^{2}+C_{\mathrm{PW}}^{2}}{C_{\mathrm{PW}}^{2}}\right)^{n}
$$

and the corresponding plug wrap paper permeability is given by

$$
\bar{K}_{\mathrm{PW}, \min }=\tilde{K}_{\mathrm{tot}}\left(\frac{C_{\mathrm{TP}}^{2}+C_{\mathrm{PW}}^{2}}{C_{\mathrm{TP}}^{2}}\right)^{n}
$$




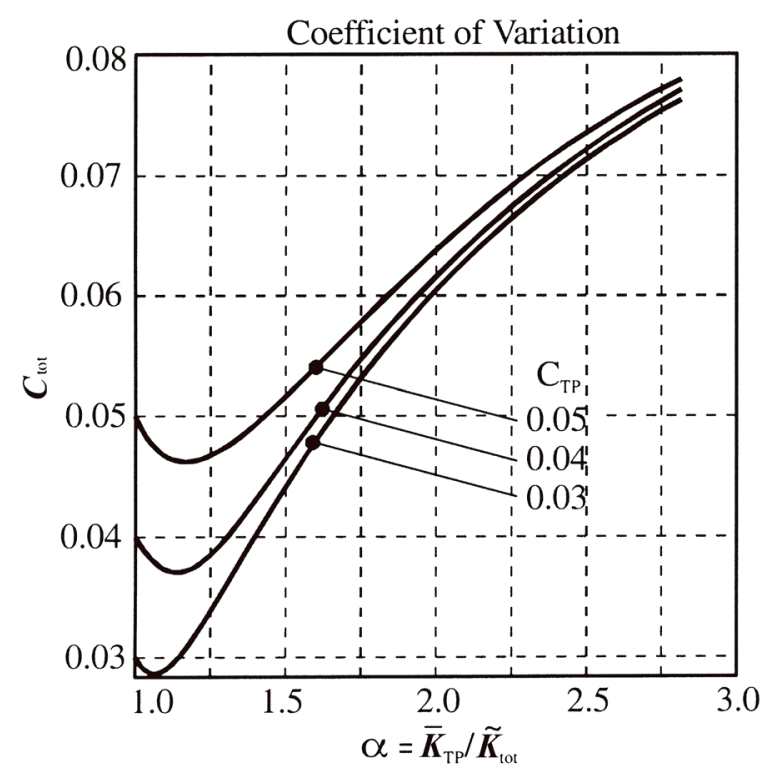

Figure 3. The coefficient of variation of the permeability of a layered structure composed of perforated tipping paper and porous plug wrap paper as a function of the ratio of the tipping permeability $\bar{K}_{\mathrm{TP}}$ to the total permeability $\tilde{K}_{\text {tot }}$. The parameter $C_{\mathrm{TP}}$ is the coefficient of variation of the tipping paper permeability.

The minimal coefficient of variation for the total permeability achieved for this tipping/plug wrap combination is

$$
C_{\text {tot }, \min }=\frac{C_{\mathrm{TP}}+C_{\mathrm{PW}}}{\sqrt{C_{\mathrm{TP}}^{2}+C_{\mathrm{PW}}^{2}}} .
$$

To illustrate the results, Figure 3 displays $C_{\text {tot }}$ as a function of $\alpha$ with $n=1$ and $C_{\mathrm{PW}}=0.12$ for various values of $C_{\mathrm{TP}}$. As can be clearly seen, a minimal coefficient of variation can be achieved if the tipping paper permeability is chosen between $10 \%$ and $50 \%$ higher than the desired total permeability. Figure 4 shows the coefficient of variation as a function of $\alpha$, this time with the exponent $n$ as a parameter, $C_{\mathrm{TP}}=0.05$ and $C_{\mathrm{PW}}=0.12$. While the minimizing $\alpha$ depends on the exponent, the minimal coefficient of variation does not depend on it.

\section{RESULTS}

The main results are Eqns. [7], [10], [12] and [14]-[16]. They will be summarized below, by demonstrating how they can be used for a real cigarette design.

We assume that from a cigarette model and our design goals with respect to smoke yields we have determined a desired total permeability (as measured according to ISO 2965) $k_{\text {tot }}=150 \mathrm{CU}$. We want to realize this permeability with an electro-perforated tipping paper with a $w=3 \mathrm{~mm}$ wide perforation zone and an exponent of $n=0.87$. The coefficient of variation for the tipping paper permeability is known to be about $C_{\mathrm{TP}, m} \approx 0.05$, so by Eqn. [8] we set $C_{\mathrm{TP}} \approx$ 0.05 . For the plug wrap paper permeability we have a coefficient of variation of $C_{\mathrm{PW}, m} \approx 0.07$, thus by Eqn. [9] $C_{\mathrm{PW}}=0.12$. Correcting for the perforation width we determine $\tilde{K}_{\text {tot }}=(10 / w) k_{\text {tot }, m}=500 \mathrm{CU}$. Due to the upper

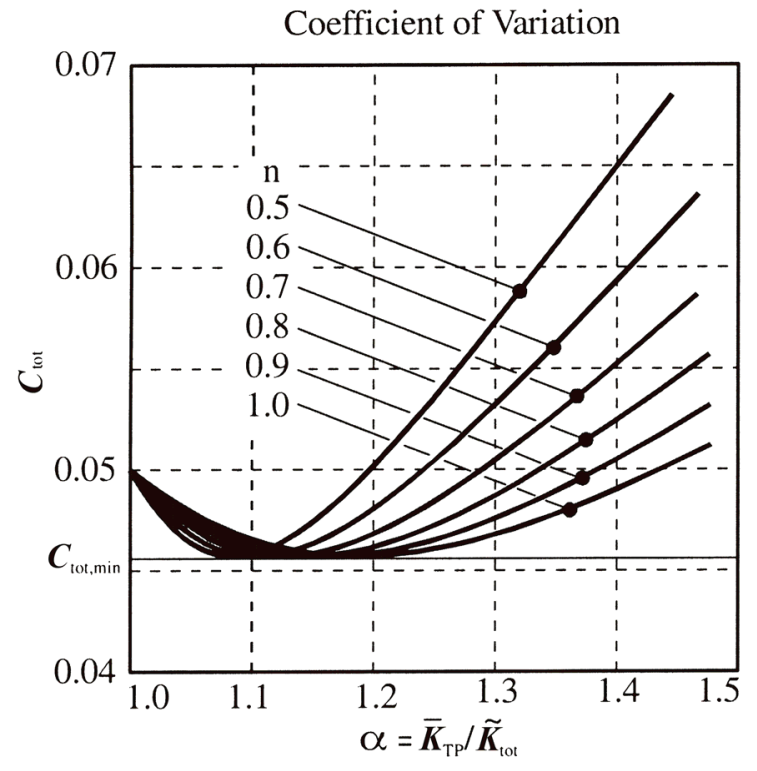

Figure 4. The coefficient of variation of the permeability of a layered structure composed of perforated tipping paper and porous plug wrap paper as a function of the ratio of the tipping permeability $\bar{K}_{\mathrm{TP}}$ to the total permeability $\tilde{K}_{\text {tot }}$. The parameter $n$ is the exponent in the non-linear pressure drop-flow relation of the layered structure.

bound derived from Eqn. [10], we may identify the expected total permeability with the total permeability calculated from the expected values of tipping and plug wrap paper permeability. Then we can directly evaluate Eqn. [14] and get the permeability of the tipping paper

$$
k_{\mathrm{TP}, m}=\frac{w}{10} \bar{K}_{\mathrm{TP}}=\frac{w}{10} 574.72 \mathrm{CU}
$$

The plug wrap paper permeability can be calculated by Eqn. [15]

$$
k_{\mathrm{PW}, m}=\bar{K}_{\mathrm{PW}}=2636.5 \mathrm{CU} \text {. }
$$

By Eqn. [16] the minimal coefficient of variation is $C_{\text {tot, min }}=0.04615$, which is lower than the coefficient of variation of the tipping paper permeability! Practically, a plug wrap paper with $2636.5 \mathrm{CU}$ will not be available, so we choose $k_{\mathrm{PW}, \mathrm{m}}=2400 \mathrm{CU}$ and correct the tipping paper permeability according to Eqn. [7] and get a final value of $k_{\mathrm{TP}, m}=$ 175.4 CU. For these values we obtain, by Eqn. [12], a coefficient of variation for the total permeability of $C_{\text {tot }}=0.04620$ which is very close to the minimal coefficient of variation.

\section{DISCUSSION}

There are several points to be noted about these equations. First of all in Eqn. [10] we demonstrate that the calculation of permeabilities can be done with their expected values, because the deviation caused by the non-linearity of Eqn. [5] is sufficiently small. This greatly simplifies the design. Furthermore, for practical purposes, the total permeability can be considered as normally distributed, which means that its statistical properties are completely determined by expected value and variance. 
From Eqn. [16], we learn that the minimal coefficient of variation is always lower than the coefficient of variation of the plug wrap paper permeability and also of the tipping paper permeability, which is quite a surprising result. It is beneficial that the minimal coefficient of variation is rather stable with respect to small variations in the expected values of tipping and plug wrap paper permeability. This increases the practical usefulness because plug wrap papers are usually not manufactured at arbitrarily chosen permeability values.

Additionally, the minimal coefficient of variation can be achieved with tipping paper permeabilities which are $10 \%$ to $50 \%$ higher than the total permeability. This agrees well with common cigarette designs and thus an optimal design will not deviate too much from existing designs.

It is also interesting to note that the minimal coefficient of variation does not depend on the chosen total permeability and on the exponent of the pressure drop-flow relationship. This also stabilizes the optimality of the design.

As a possible extension of this study the distance between tipping and plug wrap due to the adhesive layer can be accounted for, by dropping the assumption $A_{\mathrm{TP}}=A_{\mathrm{PW}}$ and repeating the derivations.

While every effort has been taken to verify the assumptions on which the model and its conclusions are based, it might also be desirable to directly verify the conclusions by experimental data. However, to detect a reduction of the coefficient of variation in the order of $0.2 \%$, for example from $5 \%$ to $4.8 \%$ at a reasonable level of statistical significance, a very high number ( $>1500)$ of measurements is necessary. Additionally, the variance inherent to the measurement device and due to the experimental procedure make this investigation rather difficult. Nonetheless, considering the good fit of the model to experimental data and the statistical evidence supporting the underlying assumptions, it seems reasonable to also trust the conclusions.

\section{REFERENCES}

1. Gaißer, H.: Die stationäre Strömung in der Cigarette und deren Einfluß auf den Zugwiderstand; Beitr. Tabakforsch. Int. 14 (1992) 93-122.

2. Dwyer, R.W. and P. Chen: Prediction of pressure drop and ventilation in a lit Cigarette; Beitr. Tabakforsch. Int. 18 (1999) 205-211.
3. Eitzinger, B. and G. Ederer: The use of nonlinear constitutive equations to evaluate draw resistance and filter ventilation; Beitr. Tabakforsch. Int. 19 (2001) 177-188.

4. Dwyer, R.W., P. Chen, and R.D. Wasyk: A mathematical scheme for calculating flows and pressure drops in lit and unlit cigarettes; Beitr. Tabakforsch. Int. 19 (2001) 189-203.

5. Schneider, W., A. Schlüter, and F. Seehofer: The effects of the properties of materials in a cigarette on filter ventilation; Beitr. Tabakforsch. Int. 12 (1984) 123-136.

6. Mathis, D.E.: Flow rate dependence of ventilation; Beitr. Tabakforsch. Int. 14 (1987) 11-19.

7. Baker, R.R.: The viscous and inertial flow of air through perforated papers; Beitr. Tabakforsch. Int. 14 (1989) 253-260.

8. Mathis, D.E.: Component analysis of pressure drop and ventilation variability; Beitr. Tabakforsch. Int. 12 (1984) 169-177.

9. ISO 2965:1997: Materials used as cigarette papers, filter plug wrap and filter joining paper, including materials having an oriented permeable zone - Determination of air permeability; International Organization for Standardization, Geneva, 1997.

10. Komatsubara, O., T. Yoshida, and K. Kaneki: Computational analysis of flow through perforations in tipping paper; Presentation at the CORESTA meeting of the Smoke Science \& Product Technology groups, Innsbruck, Abstract ST13, 1999.

11. Hartung, J.: Statistik; 11. Auflage, Oldenbourg Verlag, München, 1998.

12. Ruymgaart, P.A. and T.T. Soong: Mathematics of Kalman-Bucy filtering; 2nd edition, Springer Series in Information Sciences, Springer Verlag, New York, 1988.

13. Doob, J.L.: Measure theory, graduate texts in mathematics 143; Springer Verlag, New York, 1993.

\section{Corresponding author}

Bernhard Eitzinger

WFT Research

Fabrikstrasse 20

4050 Traun

Austria 


\section{APPENDIX}

\section{Proof of Eqns. [8] and [9]}

Let $v(x)=K(x) \cdot \Delta p^{n}$ be the flow velocity at position $x$ through an air permeable paper of the local random permeability $K(x)$. The total volumetric flow through the area $A$ is then given by

$$
\dot{V}=\int_{A} K(x) \cdot \Delta p^{n} d A
$$

Let $K(x)$ be identically distributed for all $x$ with expected value $E(K)$ and variance $V(K)$. Furthermore let $K(x)$ be independent, so that the covariance $\operatorname{COV}(K(x), K(y))=0$ for $x \neq y$. The mean permeability $K_{\mathrm{A}}$ assigned to the area $A$ is

$$
K_{\mathrm{A}}=\frac{\dot{V}}{A \Delta p^{n}}=\frac{1}{A} \int_{A} K(x) d A .
$$

Its expected value can be calculated under the above assumptions from

$$
\left.E\left(K_{\mathrm{A}}\right)=E\left(\frac{1}{A} \int_{A} K(x) d A\right)=\frac{1}{A} \int_{A} E(K(x)) d A\right)=E(K)
$$

and its variance is given by

$$
\left.V\left(K_{\mathrm{A}}\right)=V\left(\frac{1}{A} \int_{A} K(x) d A\right)=\frac{1}{A^{2}} \int_{A} V(K(x)) d A\right)=\frac{1}{A} V(K) .
$$

The above two equations are a standard result in the signal processing literature and can be found, for example in (12) Proofs and conditions for the existence of these integrals are given in (13).

Hence the coefficients of variation for two areas $A_{1}$ and $A_{2}$ are related by

$$
\sqrt{A_{1}} C_{\mathrm{A}_{1}}=\frac{\sqrt{V(K)}}{E(K)}=\sqrt{A_{2}} C_{\mathrm{A}_{2}}
$$

or

$$
C_{\mathrm{A}_{2}}=\sqrt{\frac{A_{1}}{A_{2}}} C_{\mathrm{A}_{1}} \text {, }
$$

which proves Eqns. [8] and [9].

Properties of $\mathrm{f}$

A few properties of the function

$$
z=f(x, y)=\frac{x y}{\left(x^{1 / n}+y^{1 / n}\right)^{n}}
$$

which are needed in the investigation are derived below. Note that $f(x, y)=f(y, x)$, therefore properties which hold for one variable will also hold for the other variable by swapping $x$ and $y$.

\section{First derivative}

By applying the standard rules of differentiation, which can be conveniently done by computer algebra, we obtain

$$
\begin{aligned}
\frac{\partial f}{\partial x} & =\frac{y}{\left(x^{1 / n}+y^{1 / n}\right)^{n}}\left[1-\frac{x^{1 / n}}{\left(x^{1 / n}+y^{1 / n}\right)^{n}}\right] \\
& =\frac{y^{1+/ n}}{\left(x^{1 / n}+y^{1 / n}\right)^{n+1}}
\end{aligned}
$$

\section{Second derivative}

The following immediately obvious equation will be needed in the derivation.

$$
\left(\frac{x y}{z}\right)^{1 / n}=x^{1 / n}+y^{1 / n}
$$

By computer algebra we get

$$
\begin{aligned}
\frac{\partial^{2} f}{\partial x^{2}}= & -\frac{y}{n}\left(n\left(x^{1 / n}+y^{1 / n}\right)^{-1-n} x^{1 / n-1}\right. \\
& -n\left(x^{1 / n}+y^{1 / n}\right)^{-2-n} x^{2 / n-1}+\left(x^{1 / n}+y^{1 / n}\right)^{-1-n} x^{1 / n-1} \\
& \left.-\left(x^{1 / n}+y^{1 / n}\right)^{-2-n} x^{2 / n-1}\right)
\end{aligned}
$$

This can be simplified by algebraic manipulations to

$$
\begin{aligned}
\frac{\partial^{2} f}{\partial x^{2}}= & -\frac{y}{n}\left[(-1-n)\left(x^{1 / n}+y^{1 / n}\right)^{-2-n} x^{2 / n-1}\right. \\
& \left.+(1+n) n\left(x^{1 / n}+y^{1 / n}\right)^{-1-n} x^{1-1 / n}\right] \\
= & \frac{1+n}{n} y^{1+1 / n} x^{1 / n-1}\left(x^{1 / n}+y^{1 / n}\right)^{-2-n}
\end{aligned}
$$

Now we can make use of Eqn. [18] and we get

$$
\begin{aligned}
\frac{\partial^{2} f}{\partial x^{2}} & =-\frac{1+n}{n} y^{1+1 / n} x^{1 / n-1}\left(\frac{x y}{z}\right)^{(-2-n) / n} \\
& =-\frac{1+n}{n} \frac{z^{1+2 / n}}{x^{2+1 / n} y^{1 / n}}
\end{aligned}
$$

which is the expression used in the derivation.

Upper bound on $\mathrm{f}$

Let $x>0, y>0$ and $n>0$. We want to prove

$$
f(x, y)=\frac{x y}{\left(x^{1 / n}+y^{1 / n}\right)^{n}}<x
$$

Obviously, the inequality $y^{1 / n}<x^{1 / n}+y^{1 / n}$ holds as all variables are positive. Hence $y<\left(x^{1 / n}+y^{1 / n}\right)^{n}$ and

$$
\frac{y}{\left(x^{1 / n}+y^{1 / n}\right)^{n}}<1
$$

A final multiplication by $x$ proves the desired result.

\section{Error estimate for the expected value}

To estimate the relative error in the expected value made by replacing $f$ with its second order Taylor series $\tilde{f}_{2}$, we numerically evaluate the integrals

$$
\begin{aligned}
E\left(f\left(K_{\mathrm{TP}}, K_{\mathrm{PW}}\right)\right)= & \iint f\left(k_{\mathrm{TP}}, k_{\mathrm{PW}}\right) \cdot p\left(K_{\mathrm{TP}}=k_{\mathrm{TP}}\right) \\
& \cdot p\left(K_{\mathrm{PW}}=k_{\mathrm{PW}}\right) d k_{\mathrm{TP}} d k_{\mathrm{PW}}
\end{aligned}
$$

and

$$
\begin{aligned}
E\left(\tilde{f}_{2}\left(K_{\mathrm{TP}}, K_{\mathrm{PW}}\right)\right)= & \iint \tilde{f}_{2}\left(k_{\mathrm{TP}}, k_{\mathrm{PW}}\right) \cdot p\left(K_{\mathrm{TP}}=k_{\mathrm{TP}}\right) \\
& \cdot p\left(K_{\mathrm{PW}}=k_{\mathrm{PW}}\right) d k_{\mathrm{TP}} d k_{\mathrm{PW}}
\end{aligned}
$$


over the domain $\left[\varepsilon, \bar{K}_{\mathrm{TP}}+5 \cdot \sqrt{V\left(K_{\mathrm{TP}}\right)}\right] \times\left[\varepsilon, \bar{K}_{\mathrm{PW}}+5 \cdot \sqrt{V\left(K_{\mathrm{PW}}\right)}\right]$ for a small but positive $\varepsilon$. The expression $p(X=x)$ denotes the probability that the random variable $X$ assumes the value $x$ and was calculated from the probability density function of a normal distribution. Tipping and plug wrap permeabilities were assumed to be independent random variables. Extending the domain of integration further does not noticeably change the resulting value. To get an upper bound on the relative error, inspection and plots of the involved functions $f$ and $\tilde{f}_{2}$ will reveal that within the parameter values of technical interest the error due to the approximation is largest for $n=1$. Further inspection will show that the relative error does not depend on the precise values of $\bar{K}_{\mathrm{TP}}$ and $\bar{K}_{\mathrm{PW}}$, but only on their ratio $\bar{K}_{\mathrm{TP}} / \bar{K}_{\mathrm{PW}}$ and that the error is higher for higher values of $\bar{K}_{\mathrm{TP}} / \bar{K}_{\mathrm{PW}}$. So to maximize the error within technically relevant limits we set $\bar{K}_{\mathrm{TP}} / \bar{K}_{\mathrm{PW}}=1 / 5$, $C_{\mathrm{TP}}=0.05, C_{\mathrm{PW}}=0.17, n=1$ and $\varepsilon=0.001$. For the numerical integration the function dblquad(@fun, $\mathrm{x}_{\min }, \mathrm{x}_{\max }, \mathrm{y}_{\min }, \mathrm{y}_{\max }$ ) provided by the numerical software package MATLAB was used. We obtain for the relative error

$$
\frac{E\left(f\left(K_{\mathrm{TP}}, K_{\mathrm{PW}}\right)\right)-E\left(\tilde{f}_{2}\left(K_{\mathrm{TP}}, K_{\mathrm{PW}}\right)\right)}{E\left(f\left(K_{\mathrm{TP}}, K_{\mathrm{PW}}\right)\right)}=-0.000280
$$

and therefore assume this value as a reasonable estimate of the maximum relative error.

\section{Error estimate for the variance}

To estimate the relative error in the variance due to the approximation of $f$ by its first-order Taylor series expansion $\tilde{f}_{1}$ we proceed in the same way as for the expected value. We numerically evaluate the integrals

and

$$
\begin{aligned}
V(f)= & \iint(E(f)-f)^{2} \cdot p\left(K_{\mathrm{TP}}=k_{\mathrm{TP}}\right) \\
& \cdot p\left(K_{\mathrm{PW}}=k_{\mathrm{PW}}\right) d k_{\mathrm{TP}} d k_{\mathrm{PW}}
\end{aligned}
$$

$$
\begin{aligned}
V\left(\tilde{f}_{1}\right)= & \iint\left(E(f)-\tilde{f}_{1}\right)^{2} \cdot p\left(K_{\mathrm{TP}}=k_{\mathrm{TP}}\right) \\
& \cdot p\left(K_{\mathrm{PW}}=k_{\mathrm{PW}}\right) d k_{\mathrm{TP}} d k_{\mathrm{PW}}
\end{aligned}
$$

over the same domain as above. The considerations concerning the choice of numerical values for the arguments also hold in this case and we obtain for the relative error

$$
\frac{V\left(f\left(K_{\mathrm{TP}}, K_{\mathrm{PW}}\right)\right)-V\left(\tilde{f}_{1}\left(K_{\mathrm{TP}}, K_{\mathrm{PW}}\right)\right)}{V\left(f\left(K_{\mathrm{TP}}, K_{\mathrm{PW}}\right)\right)}=-0.00441 .
$$

Therefore we assume $0.44 \%$ as an upper limit on the relative error of the estimated variance. 